\title{
HIGH ENERGY GAIN IFEL AT UCLA NEPTUNE LABORATORY
}

\author{
P. Musumeci*, S. Ya. Tochitsky, S. Boucher, A. Doyuran, R. J. England, \\ C. Joshi, C. Pellegrini, J. Ralph, J. B. Rosenzweig, C. Sung, G. Travish, R. Yoder, \\ University of California at Los Angeles, CA 90095, USA \\ A. Varfolomeev, S. Tolmachev, A. Varfolomeev Jr., T. Yarovoi, RRCKI, Moscow, Russia
}

\begin{abstract}
We report on the observation of energy gain in excess of $20 \mathrm{MeV}$ at the Inverse Free Electron Laser Accelerator experiment at the Neptune Laboratory at UCLA. A $14.5 \mathrm{MeV}$ electron beam is injected in a $50 \mathrm{~cm}$ long undulator strongly tapered both in period and field amplitude. $\mathrm{A} \mathrm{CO}_{2} 10.6 \mu \mathrm{m}$ laser with power $>400 \mathrm{GW}$ is used as the IFEL driver. The Rayleigh range of the laser $(\sim 1.8 \mathrm{~cm})$ is shorter than the undulator length so that the interaction is diffraction dominated. Few per cent of the injected particles are trapped in stable accelerating buckets and electrons with energies up to $35 \mathrm{MeV}$ are detected on the magnetic spectrometer. Three dimensional simulations are in good agreement with the electron energy spectrums observed in the experiment and indicate that substantial energy exchange between laser and electron beam only occurs in the first $25-30 \mathrm{~cm}$ of the undulator. An energy gradient of $>70 \mathrm{MeV}$ is inferred. In the second section of the undulator higher harmonic IFEL interaction is observed.
\end{abstract}

\section{INTRODUCTION}

Laser acceleration of charged particles in vacuum is of great interest since the electric field of a laser at its focus can exceed by many orders of magnitude the average acceleration gradient for a conventional radiofrequency accelerator (e.g. $50 \mathrm{MeV} / \mathrm{m}$ for a S-band accelerator), which is fundamentally limited by the field emission on the copper walls. In addition laser acceleration allows control of the electron bunch length on the scale of the laser wavelength $\lambda$. Among all proposed concepts for laser acceleration, the Inverse Free Electron Laser (IFEL) scheme is one of the most mature. In an IFEL $[1,2]$, relativistic particles are moving through an undulator magnet; a laser beam is propagating parallel to the electron beam. The undulator produces a small transverse velocity in a direction parallel to the electric vector of the electromagnetic wave so that energy can be transferred between the particles and the wave. Efficient energy exchange takes place when the electron phase slippage is such that its transverse velocity changes sign synchronously with the laser field (resonant condition).

Recent successful proof-of-principle IFEL experiments have shown that along with acceleration $[3,4]$ this scheme offers the possibility to manipulate and control the longitudinal phase space of the output beam at the laser wavelength. First among other laser accelerator schemes, the

\footnotetext{
${ }^{0}$ Now at INFN - Universita' di Roma La Sapienza
}

Inverse Free Electron Laser has in fact experimentally demonstrated microbunching [5], phase-dependent acceleration of electrons [6], phase locking and multi-stage acceleration [7] and control of final energy spread [8]. Despite such significant progress on a phase-controlled acceleration, in all these experiments the energy gain was modest and the measured acceleration gradient was well below the gradient attributed to the conventional if accelerator.

It is of great importance to demonstrate both high energy gain and acceleration gradient in a laser accelerator. This can be achieved not only by increasing the coupled laser power, but also by increasing the effective interaction length. The latter is obtained a) by extending the region where the laser is most intense (diffraction effects) and b) by increasing the length over which the particles and the laser wave maintain synchronism (tapering).

The inverse free-electron laser experiment at the Neptune laboratory at UCLA accelerated electrons from 14.5 $\mathrm{MeV}$ up to more than $35 \mathrm{MeV}$ utilizing a $\mathrm{CO}_{2}$ laser beam with a peak power $(\sim 0.4 \mathrm{TW})$, one order of magnitude greater than any other previous IFEL experiment had used [16]. To maintain the resonant condition with the accelerating electrons, the $50 \mathrm{~cm}$ long undulator is strongly tapered both in period and magnetic field amplitude. An important point of the Neptune IFEL configuration is that the Rayleigh range of the laser beam is much shorter than the undulator length and the IFEL interaction is diffraction dominated. We also report about the observation of higher harmonic IFEL interaction manifested in a modulation of the output electron energy spectrum.

\section{EXPERIMENTAL SETUP}

In Table 1 we report the design parameters of the IFEL experiment at the Neptune Laboratory.

In Fig. 1 it is shown the experimental layout for the IFEL experiment. An electron beam of $300 \mathrm{pC}$ at $14.5 \mathrm{MeV}$ is

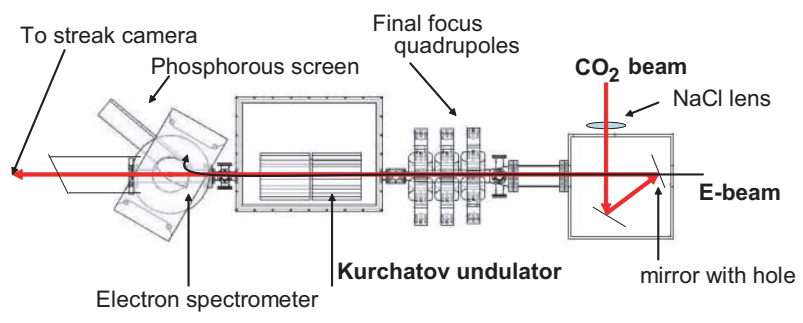

Figure 1: Layout of Neptune IFEL experiment. 
Table 1: Electron beam and $\mathrm{CO}_{2}$ Laser parameters at the Neptune Laboratory

\begin{tabular}{|l|c|}
\hline Electron beam & \\
\hline energy & $14.5 \mathrm{MeV}$ \\
charge & $0.3 \mathrm{nC}$ \\
emittance & $5 \mathrm{~mm}-\mathrm{mrad}$ \\
pulse length (rms) & $6 \mathrm{ps}$ \\
$\sigma_{r m s}$ at focus & $120 \mu \mathrm{m}$ \\
\hline $\mathrm{CO}_{2}$ laser & \\
\hline power & $400 \mathrm{GW}$ \\
wavelength & $10.6 \mu \mathrm{m}$ \\
pulse length (FWHM) & $240 \mathrm{ps}$ \\
spot size $\left(1 / \mathrm{e}^{2}\right)$ & $240 \mu \mathrm{m}$ \\
\hline
\end{tabular}

delivered to the experimental region by the Neptune rf photoinjector [9]. Final focus quadrupoles with large aperture to avoid clipping of the copropagating laser beam were installed on the beamline and focused the electron beam to the nominal spot size of $120 \mu \mathrm{m} \mathrm{rms}$ in the middle of the undulator. A TW-class $\mathrm{CO}_{2}$ laser system [10] is used to drive the IFEL. The high power laser beam is brought in vacuum through a $\mathrm{NaCl}$ lens that has both the function of producing the correct focusing geometry and serves as a vacuum window. The laser is made collinear to the e-beam utilizing a plane copper mirror with a hole. The beams are aligned on a detection screen in the midplane of the undulator with an accuracy of $<100 \mu \mathrm{m}$ and the angular misalignment is kept below $1 \mathrm{mrad}$ using the screens located before and after the undulator. After the interaction region, the e-beam is energy analyzed by the magnetic spectrometer and the laser beam is sent to a streak camera for timing measurements.

The IFEL hybrid planar undulator KIAE-2p $[11,12]$ was designed and built at the Kurchatov Institute. This magnet was optimized for a $400 \mathrm{GW}, 3.6 \mathrm{~cm}$ Rayleigh range driver laser beam. It has a very strong variation of the wiggling period and magnetic field amplitude along the axis (see Table 2), carefully tailored to maintain the resonance condition of the IFEL interaction between the $\mathrm{CO}_{2}$ photons and the quickly accelerating electrons. Because the Rayleigh range is much shorter than the undulator length, the IFELdriving laser field along the undulator varies rapidly, and it becomes important to properly consider the Guoy phase shift experienced by the laser near the focal point [13]. In order to maintain synchronism through the focal region, two undulator sections were built with the field in the central region adjusted using a corrector Vanadium-Permandur plate. The undulator period and field strength were specified using simulation results [11] obtained with the three dimensional code TREDI [14].

Synchronization between electrons and photons is the key for every laser accelerator with an externally injected electron beam and it is even more delicate in an IFEL experiment. In this kind of interaction in fact, there is no resonant medium (or cavity) where the accelerating electromagnetic wave is resonating and can live independently

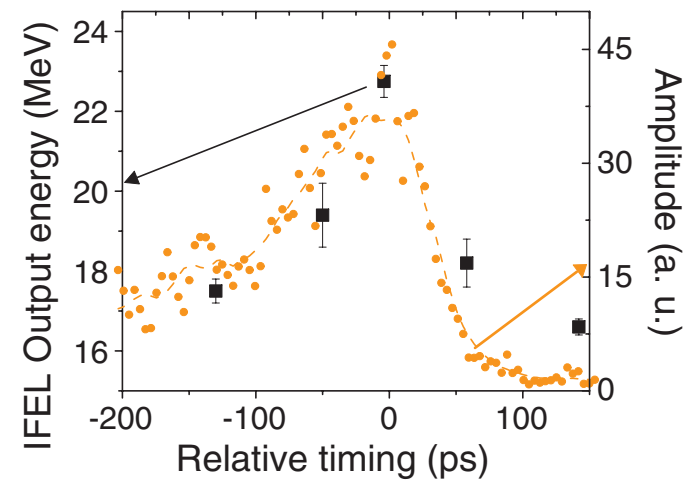

Figure 2: IFEL output energy vs. measured delay on the streak camera between reference e-beam pulse and $\mathrm{CO}_{2}$ laser. Also shown is a typical streak of the $\mathrm{CO}_{2}$ laser pulse.

than the laser beam. The accelerator is virtually turned on only at the time the laser pulse goes through the undulator. The ponderomotive gradient that accelerates the electrons depends on the instantaneous power felt by the electrons.

At the Neptune laboratory, the electron pulse $(\sim 15 \mathrm{ps}$ FWHM) and the longer $\mathrm{CO}_{2}$ laser pulse $(\sim 240 \mathrm{ps} \mathrm{FWHM})$ have been synchronized in the past with a cross-correlation method that was based on the e-beam controlled transmission of $\mathrm{CO}_{2}$ through a Germanium sample. This technique [15] constituted the first step of the synchronization procedure also in the IFEL experiment. On the other hand, the cross-correlation measurement is conducted with the unamplified laser pulse propagating through the final triple pass, $2.5 \mathrm{~m}$ long multiatmosphere $\mathrm{CO}_{2}$ amplifier with no inversion of population and has an intrinsic systematic error due to the different group velocity of the laser pulse within the inverted medium of the final amplifier in comparison with no-gain conditions. Moreover, fluctuations in laser power (gain in the final amplifier) $\pm 50 \%$ cause fluctuations in the time of arrival of $\pm 50 \mathrm{ps}$. This jitter is intrinsic in the laser amplification system and could not be eliminated.

In order to get very accurate information on the relative timing between the electrons and the amplified pulse on each shot, we set up a new streak camera based timing diagnostics. The cathode of the streak camera is not sensitive to $10.6 \mu \mathrm{m}$ photons so an optical gating technique, based on the Kerr effect, was used to gate a long red laser diode with the $\mathrm{CO}_{2}$ pulse. This gated portion of red pulse is sent on the entrance slit of a 10 ps resolution streak camera together with a small fraction of the photocathode driver laser

Table 2: RRCKI IFEL undulator parameters

\begin{tabular}{c|cc}
\hline & initial & final \\
\hline Undulator period & $1.5 \mathrm{~cm}$ & $5 \mathrm{~cm}$ \\
Magnetic field amplitude & $0.16 \mathrm{~T}$ & $0.65 \mathrm{~T}$ \\
$\mathrm{~K}$ & 0.2 & 2.8 \\
Resonant energy & $14.5 \mathrm{MeV}$ & $52 \mathrm{MeV}$ \\
\hline
\end{tabular}


as reference for the electron beam timing. In Fig. 2, the final maximum energy of the IFEL accelerator is shown as a function of delay between the e-beam reference pulse and the peak of the $\mathrm{CO}_{2}$ pulse, as measured on the streak camera. A lineout of a typical $\mathrm{CO}_{2}$ pulse is also displayed on the same time scale. Even for very small laser power some interaction is observed $(+150$ ps data point). On the other hand, the output energy is clearly maximized when the electrons sample the peak laser intensity. This diagnostic allowed us to determine for each laser shot the pulse length -and thus the peak power- of the $\mathrm{CO}_{2}$ beam and which part of the laser pulse intensity profile the electron beam sampled with an accuracy of $\pm 10 \mathrm{ps}$. This measurement was used to optimize the injection time of the electrons in the IFEL accelerator.

The optical geometry used in the experiment to focus and control transversely the laser beam size is of particular importance. In the original design, a $2.56 \mathrm{~m}$ focal length $\mathrm{NaCl}$ lens focused the laser in the middle of the undulator to a spot size of $340 \mu \mathrm{m}$ with a Rayleigh range of $3.6 \mathrm{~cm}$ to increase as much as possible the extent of the region where the beam is more intense. The resulting peak intensity is $2 \cdot 10^{14} \mathrm{~W} / \mathrm{cm}^{2}$ in the laser focus, about two orders of magnitude more than any previous IFEL experiment used [7, 8]. Dealing with this very high laser intensities on the other hand had some disadvantages. Experimentally, in fact, we were limited by damage threshold on the last optical elements of the $\mathrm{CO}_{2}$ transport line and we could not increase the f-number of the optical geometry as we planned. For our typical pulse lengths of $200 \mathrm{ps}$, we observed damage on the copper mirrors for fluences above $3 \mathrm{~J} / \mathrm{cm}^{2}$ and on the single crystal $\mathrm{NaCl}$ optics for fluences above $2 \mathrm{~J} / \mathrm{cm}^{2}$. In the end, we measured a spot size of $240 \mu \mathrm{m}$ and Rayleigh range of $1.8 \mathrm{~cm}$ that, respect to the original design geometry, implied a stronger variation of the beam size along the interaction region, and with the same nominal focus position at the mid-point of the undulator, a larger and less intense beam at the entrance and exit of the undulator.

To trap and accelerate particles along the design resonant orbit, the ponderomotive IFEL gradient generated by the laser electric field has to match the designed tapering gradient. If this is not the case, no trapping or acceleration are possible. Because of the differences between nominal and measured Rayleigh range, it was found out necessary to move the laser focus upstream from the nominal position to increase the intensity at the undulator entrance above the trapping threshold and start the acceleration process early in the undulator. Of course that would also cause the particles to fall out of resonance soon after the mid point of the undulator because of lack of enough ponderomotive force to sustain the acceleration along the designed orbit. Positioning the laser focus $2 \mathrm{~cm}$ ( $\sim$ one Rayleigh range) upstream of the undulator midpoint was found to give the maximum final energy. When moving the focus towards the undulator center, the intensity at the magnet entrance drops below the trapping threshold and there is no resonant IFEL interaction. If the laser focus is moved further up-

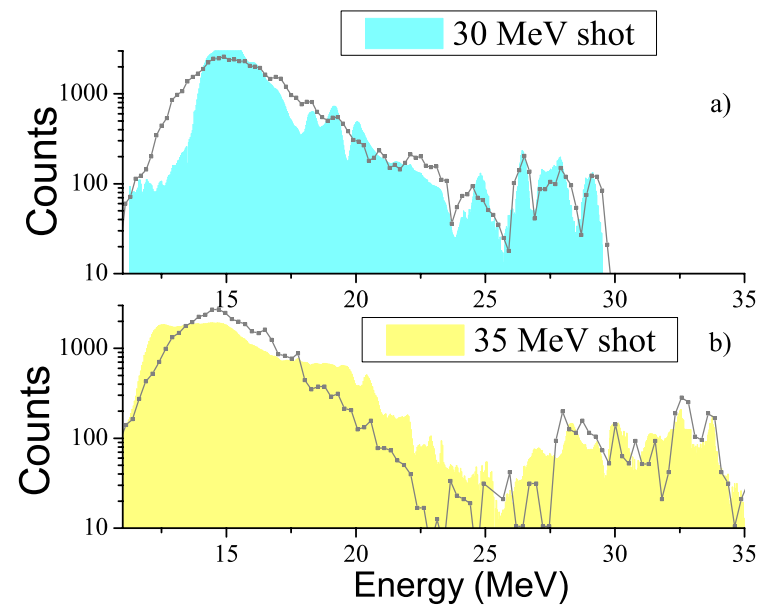

Figure 3: Measured and simulated output energy spectrums for the Neptune IFEL accelerator experiment for two different laser focus positions. The simulated spectra have been normalized to the same area as the measured ones.

stream from the optimum point, the final energy decreases because the effective interaction length is shortened.

\section{EXPERIMENTAL RESULTS AND SIMULATIONS}

The electrons were detected by a phosphorous screen observed by CCD cameras. The screen was attached to the thin mylar window at the exit slit of the magnetic broadband spectrometer. Different neutral density filters were applied to each cameras to get unsaturated images of the output slit. A postprocessing application that takes into account the different filters and scales the horizontal axis of the images with energy, reconstructed the single shot spectrum of the electron beam out of the IFEL accelerator for each laser shot. In Fig. 3 we show two measured energy spectra (histograms in Fig. 3) obtained with the laser focus at two different position, respectively $5 \mathrm{~cm}$ and $2 \mathrm{~cm}$ upstream of the undulator midpoint. The laser power for these shots was $350 \mathrm{GW}$ and $400 \mathrm{GW}$, respectively. As discussed earlier, when focusing the laser beam earlier along the undulator, less power is required to trap and accelerate the electrons, but their final energy is lower because the interaction length is reduced by the detrapping occurring when the laser intensity falls below the trapping threshold value.

The energy spectrum in Fig. 3b shows more than $5 \%$ of particles trapped and accelerated up to $35 \mathrm{MeV}$ with a $150 \%$ energy gain. The dispersion $\delta=\Delta x / \Delta p$ is not constant along the measurement screen, and it grows from $1 \mathrm{~cm} / \mathrm{MeV}$ for the lower energies end to $4 \mathrm{~cm} / \mathrm{MeV}$ for higher energies. The resolution is sufficient to fully distinguish different peaks in the high energy part of the spectra. The camera noise level sets a minimum detectable charge density. Converting this threshold into the graph units, we obtain a minimum detection threshold below 10 counts. 

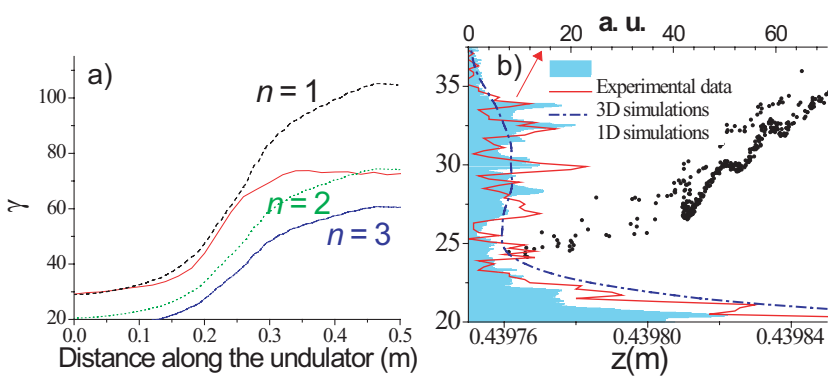

Figure 4: Simulations of IFEL experiment. (a) Maximum energy particle evolution along the undulator. The first, second and third harmonic resonant curved are also shown. (b) Longitudinal phase space at $45 \mathrm{~cm}$ with the energy modulation due to the higher harmonic interaction

Adjusting the laser intensity distribution along the undulator to the experimental configuration, the simulation results agree quite well with the experiment. The code that was used to simulate the experiment is a 4th-order Runge-Kutta integrator that solved the Lorentz equation for the particle motion in the combined fields of the undulator magnet and laser beam (TREDI[14]).

In Fig. 4a we show the evolution of the maximum ebeam energy along the accelerator for the case where the laser focus is located $2 \mathrm{~cm}$ upstream of the undulator midpoint (solid red line in Fig. 4a). The IFEL acceleration mostly takes place in the first section of the undulator (first $25-30 \mathrm{~cm}$ ). This gives an accelerating gradient larger than $70 \mathrm{MeV} / \mathrm{m}$ in the first half of the undulator. Few $\mathrm{cm}$ after the mid-point the laser intensity has decreased below the trapping threshold and the designed tapering is too strong for the particles too follow.

Fig. $4 \mathrm{~b}$ shows the simulated longitudinal phase-space at $45 \mathrm{~cm}$ along the undulator. The solid red line on the left of the graph is the projection of the phase-space on the energy axis. Also shown is the measured experimental spectrum (cyan histogram). The energy modulation appearing in both the simulated and experimental energy spectra is particularly interesting because the spectrum predicted by the solution of the 1D FEL-like equations does not show any peaks (blue dotted line). The high energy side of the 3D simulation spectrum, a few $\mathrm{cm}$ after the midpoint, develops a peaked structure similar to that observed in the data. Experimentally, this structure was reproducible shot to shot, ruling out the possibility of being caused by microstructures present in the e-beam or in the laser beam.

The IFEL resonance condition is ordinarily understood to mean that efficient energy exchange between the transverse EM wave and the electrons can only take place at electron energies such that, in the electron rest frame, the wiggling induced by the laser has the same frequency as the wiggling induced by the undulator. However, when the motion in the electron rest frame is not a simple dipole oscillation, resonance can also occur if the laser frequency is a multiple of the undulator wiggling frequency, that is, electrons of a fixed energy may interact not only with the fun- damental radiation frequency but also with its harmonics [18]. From an alternative point of view, particles of different energies $\gamma_{r, n}$ can interact with the same laser frequency, because they see the EM wave as a higher harmonic of the fundamental frequency which they are resonant with. In other words, for a given laser wavelength and undulator magnet, there are multiple resonant energies

$$
\gamma_{r, n}=\sqrt{\frac{\lambda_{w}\left(1+K^{2} / 2\right)}{2 n \lambda}}
$$

where $\lambda_{w}$ is the undulator period, $K$ is the normalized undulator amplitude and $\lambda$ the radiation wavelength. The higher harmonic IFEL interaction can be viewed as the natural consequence of the fact that the spectrum of the spontaneous radiation emitted by a relativistic electron passing through a planar undulator magnet presents peaks at different frequencies that are harmonics of a fundamental resonant frequency. In a quantum mechanic description of the interaction, because of the presence of strong resonant lines in the spontaneous radiation spectrum, stimulated emission (FEL emission at higher harmonics) or absorption (higher harmonic IFEL) of radiation at these frequencies is possible.

To explain the structure of the high energy side of the experimental spectrum in the Neptune IFEL experiment, we consider the evolution of the maximum-energy particle in the simulations represented in Fig. 4a. A few $\mathrm{cm}$ after the undulator center, the particles fall off the resonance curve (first harmonic resonance, $n=1$ dashed curve in Fig. 4a) because the driving laser intensity has decreased below the trapping threshold. Some distance later, their energy is a factor of $\sqrt{2}$ less than the first harmonic resonant energy at that point of the undulator, and therefore the electrons are resonant with the $n=2$ dotted curve and can exchange energy with the $10.6 \mu \mathrm{m}$ photons via the second harmonic IFEL interaction. Later on, the electrons have energies such that energy exchange with the laser can start even via the stronger third harmonic coupling ( $n=3$ dash-dotted curve).

It is known that the on-axis spectral content of the planar undulator radiation presents peaks only at the odd harmonics. The even harmonics in fact, are suppressed by the symmetry of the radiation emitting source. Only off-axis, the even harmonic radiation is present. Correspondingly, in the IFEL interaction, the strength of the interaction between the relativistic electrons and the even-harmonics radiation also vanishes for a perfectly symmetric interaction. Different factors contribute to the second harmonic coupling observed in the Neptune IFEL experiment. This experiment was characterized by the fact that the laser beam was coupled in the undulator with a Rayleigh range short compared to the undulator length. Thus three-dimensional effects dominated the dynamics of the IFEL interaction. In particular, the laser waves have an intrinsically large angular spread characterized by the diffraction angle. Unavoidable angular misalignments, as well as trajectory offsets of the electron and the laser beams, possibly contributed to the second harmonic interaction coefficient. Moreover, the 
laser mode used in the experiment is not a purely Gaussian $\mathrm{TEM}_{00}$ mode, as it was assumed in the design and in the simulations. After passing in the final amplifier, the transverse profile of the $\mathrm{CO}_{2}$ pulse experimentally shows a supersaturated Gaussian profile, with a content of different higher order Hermite-Gaussian modes.

The higher harmonic interaction observed in the Neptune IFEL experiment is weak, but the energy exchange is significant because the laser is still somewhat intense $\left(>10^{10} \mathrm{~W} / \mathrm{cm}^{2}\right)$ and the wiggling parameter $K$ is large at the end of the undulator. A small energy exchange takes place, enough to modulate the final energy distribution and this corresponds to the peaked microstructure observed reproducibly in the experimental spectrum.

\section{CONCLUSION}

We report on the observation of $>20 \mathrm{MeV}$ energy gain $(150 \%)$ at the Inverse Free Electron Laser experiment at the Neptune Laboratory. An energy gradient of $>70$ $\mathrm{MeV} / \mathrm{m}$ is inferred. The fraction of self-trapped particles exceeded $5 \%$ of the injected bunch. The acceleration gain reported is to date the highest obtained with an IFEL accelerator. Self-trapping of particles in a stable accelerating bucket from a not-prebunched initial distribution was demonstrated. The effects of the laser diffraction were analyzed in the design phase and studied experimentally. Finally, for the first time higher harmonic IFEL (HH-IFEL) interaction was observed in the second section of the undulator. The HH-IFEL adds a degree of freedom (the harmonic coupling number $n$ ) in the design of magnetic systems capable of coupling lasers and electron beams. This work is supported by U.S. Dept. of Energy grant DE-FG0392ER40693

\section{APPENDIX}

In the Neptune IFEL experiment, the choice of the optical scheme was constrained by the space available in the experimental hall. In theory one can try to answer the question: what is the optimum Rayleigh range for an inverse free-electron laser accelerator given a certain amount of laser power available? What is the best optical scheme to maximize the exchange of energy between the photons and the electrons?

The optimum point is a result of the compromise of focusing tighter to increase the intensity and keep the beam size uniform along the undulator to minimize the diffraction effects. To find it, keeping the focal point of the laser in the center of the undulator $\left(z_{w}=L_{u} / 2\right)$, we should maximize the available gradient integrated along the undulator. The available gradient is proportional to $k K_{l}(z) K$ where $K_{l}$ is the normalized vector potential of the electromagnetic wave, and for a gaussian laser beam with power
$P$ freely propagating in vacuum we can write

$$
\frac{\partial \gamma}{\partial z} \propto \sqrt{\frac{P}{\lambda z_{r}} \cdot \frac{1}{1+\left[1+\left(\frac{z-\frac{L_{u}}{2}}{z_{r}}\right)^{2}\right]}}
$$

For a constant K IFEL, the optimum Rayleigh range is found by imposing

$$
\frac{\partial}{\partial z_{r}} \int_{0}^{L_{u}}\left[\frac{P}{\lambda z_{r}} \cdot \frac{1}{1+\left[1+\left(\frac{z-\frac{L_{u}}{2}}{z_{r}}\right)^{2}\right]}\right]^{1 / 2} d z=0
$$

whose solution (numerically obtained) is

$$
\frac{z_{r}}{L_{u}}=0.15 \text {. }
$$

If $K$ grows along the undulator as it would be the case in a strongly tapered undulator, we have to solve

$$
\frac{\partial}{\partial z_{r}} \int_{0}^{L_{u}}\left[\frac{P}{\lambda \cdot z_{r}} \cdot \frac{K(z)}{1+\left[1+\left(\frac{z-\frac{L_{u}}{2}}{z_{r}}\right)^{2}\right]}\right]^{1 / 2} d z=0
$$

and the optimum ratio depends on how fast the undulator is tapered. For $K(z)$ growing polinomially along the undulator the optimum ratio is in the range 0.15-0.25.

The conclusion is that for given input power $P$, the best coupling of a diffraction dominated laser beam and an electron beam in a IFEL accelerator is given by a choice of the Rayleigh range about one fifth of the undulator length.

\section{REFERENCES}

[1] R.B. Palmer. J. Applied Physics, 43:3014, 1972.

[2] E. D. Courant, C. Pellegrini, and W. Zakowicz. Phys. Rev. A, 32:2813, 1985.

[3] I. Wernick and T. C. Marshall. Phys. Rev. A, 46:3566, 1992.

[4] A. Van Steenbergen et al. Phys. Rev. Lett., 77:2690, 1996.

[5] Y. Liu et al. Phys. Rev. Lett., 80:4418, 1998.

[6] R. B. Yoder, T. C. Marshall, and J. L. Hirshfield. Phys. Rev. Lett., 86:1765, 2001.

[7] W. Kimura et al. Phys. Rev. Lett., 86:4041, 2001.

[8] W. Kimura et al. Phys. Rev. Lett., 92:054801, 2004.

[9] S. G. Anderson et al. AIP Conf. Proc., 569:487, 2000.

[10] S. Ya. Tochitsky et al. Opt. Lett., 24:1717, 1999.

[11] A. A. Varfolomeev et al., NIM A, 483:377, 2002.

[12] S. Tolmachev et al. Proc. of 2004 FEL conference, Trieste, Italy, 504 (2004)

[13] P. Musumeci et al. AIP Conf. Proc., 647:278, 2003.

[14] L. Giannessi, P. Musumeci, M. Quattromini Nucl. Instr. Meth. A, 436:443, 1999.

[15] S. Ya. Tochitsky et al. Phys. of Plasmas, 11:2875, 2004.

[16] P. Musumeci et al. Phys. Rev. Lett., 94:154801, 2005.

[17] M. J. Schmidt and C. J. Elliot Phys. Rev. A, 34:4843, 1986.

[18] P. Musumeci et al. 2005. accepted for publication in PRE. 